\title{
UM PROJ ETO INTERDISCIPLINAR DE CONTROLE DE INFECÇÕES HOSPITALARES - PASSOS PARA A IMPLANTAÇÃO E POSSÍVEIS DESDOBRAMENTOS
}

\author{
An Interdisciplinary Project of Nosocomial Infections Control - \\ Steps to the implantation and possible unfoldings \\ Un Proyecto Interdisciplinar de Control de las Infecciones Hospitalarias - \\ Pasos para la implantación y posibles desdoblamientos
}

Maria Cristina Soares Rodrigues

\begin{abstract}
Resumo
Este trabalho apresenta um projeto de extensão de enfermagem no controle de infecções hospitalares, desenvolvido em um hospital-escola. Tem como pressupostos a interação ensino-pesquisa-extensão e a integração universidade-comunidade. Trata-se de um relato de experiência que parte das bases teóricas que subsidiaram a elaboração da proposta, seguido da descrição dos objetivos da ação extensionista e dos agentes de atuação, o cenário da prática, as etapas de operacionalização e o sistema de avaliação do projeto, indicando seus componentes metodológicos e o financiamento da proposta. Os resultados nessa fase inicial de desenvolvimento do projeto apontam a necessidade de avanço e de inovação das atividades de ação contínua de enfermagem na vigilância de infecções hospitalares. Assim, essa experiência integradora e interdisciplinar contribuiu para a formação do futuro profissional enfermeiro (a), abriu canais de interlocução para o enriquecimento recíproco e estreitou a relação entre trabalho acadêmico e assistência à saúde.
\end{abstract}

Palavras-chave: Infecção Hospitalar. Controle de Infecções. Relações Interprofissionais. Enfermagem.

\begin{abstract}
This work presents a nursing extension project about the control of cross infection development in a school hospital. It has as purposes the interaction between educationstudies-extension and the integration between university and community. It is a report of an experience starting from the theoretical basis which fomented the elaboration of the proposal, followed by the description of extensionist action's purposes and its agents, the scenery of practice, the operation stages, the project evaluation system which indicates its methodological components and the proposal's financing. The results in this initial phase of the project's development point to the necessity to advance and innovate the activities of nursing continuous action on the nosocomial infections' vigilance. Thus, this integrator and interdisciplinary experience had contributed to the formation of future nursing professionals, opened communication channels for a reciprocal improvement and narrowed the relations between academic work and health assistance.
\end{abstract}

\begin{abstract}
Resumen
Este trabajo presenta un proyecto de extensión de enfermería en el control de infecciones hospitalarias desarrollo en un hospital-escuela. Tiene como presupuestos la interacción entre enseñanza-investigación-extensión y la integración entre universidad - comunidad. Se trata de un relato de experiencia partiendo de las bases teóricas que animaron la elaboración de la propuesta, seguida de la descripción de los objetivos de la acción de extensión y de los agentes de actuación, el escenario de la práctica, las etapas de la operacionalización, el sistema de evaluación del proyecto indicando sus componentes metodológicos y la financiación de la propuesta. Los resultados en esta etapa inicial de desarrollo del proyecto indican la necesidad de avanzar e de innovación de las actividades de acción continua de enfermería en la vigilancia de infecciones hospitalarias. Así, esa experiencia integradora e interdisciplinaria contribuyó para la formación del futuro profesional enfermero(a), abrió cauces de interlocución para el enriquecimiento recíproco y estrechó la relación entre trabajo académico y asistencia a la salud.
\end{abstract}

Keywords: Cross Infection. Infection Control. Palabras clave: Infección Hospitalaria. Control de Interprofessional Relations. Nursing. 


\section{INTRODUÇÃO}

A sociedade do século XXI vivencia a situação do acréscimo populacional, as limitações das fontes de recursos e a demanda excessiva por educação'. No que se refere à educação superior, a Universidade brasileira é conclamada a redefinir estratégias que the possibilite sobreviver e atender às suas finalidades, vivenciando um momento de discussão e reflexão, na perspectiva da reforma universitária.

Considerando os desafios pelos quais as Instituições Federais de Ensino Superior (IFES) passam - a busca da qualidade científica, tecnológica e artístico-cultural e de interação com a sociedade por meio de ações de promoção e garantia de valores democráticos de igualdade e desenvolvimento social - e levando em conta o perfil acadêmico e o papel social, é que se legitima a extensão universitária, como processo orgânico e contínuo do trabalho acadêmico?

No cerne desses pressupostos, a extensão universitária é concebida como o processo educativo, cultural e científico que articula o ensino e a pesquisa de forma indissociável e viabiliza a relação transformadora entre universidade e sociedade ${ }^{3}$. Esta concepção se baseia na premissa do compromisso social da Universidade, enquanto instituição empenhada no equacionamento de questões que afligem a sociedade. Desse modo, a extensão universitária constitui elemento capaz de operacionalizar a relação teoria/prática, promovendo a aprendizagem recíproca entre alunos, professores e comunidade envolvida.

A partir dessas diretrizes norteadoras, as ações de extensão realizadas pela Universidade de Brasília (UnB), e que são viabilizadas pelo Decanato de Extensão (DEX), têm o propósito precípuo de promover a interação transformadora entre a instituição e a sociedade, integrando as artes e a ciência ao ensino, à pesquisa e ao desenvolvimento social ${ }^{4}$.

De acordo com a política de extensão em vigor na UnB, as ações de extensão apresentam grande diversidade e derivam da natureza da instituição, cuja função é cultivar o saber, no sentido de sua produção, disseminação e aplicação ${ }^{4}$. Assim, diferentes projetos de extensão são conduzidos e refletidos pela comunidade acadêmica da UnB, indo ao encontro das necessidades das diversas comunidades que integram o Distrito Federal (DF).

Em 2004 foi implantado o Projeto de Extensão 'Atuação de Enfermagem no Programa de Controle de Infecção Hospitalar do Hospital Universitário de Brasília'. A proposta foi idealizada a partir do interesse de uma professora do Curso de Enfermagem da instituição com o propósito de tornar oportuno o saber-fazer e saberser enfermeiro (a) na prevenção e controle de infecções hospitalares, por meio de ação extensionista. Esta intenção se fundamentava no fato de que havia uma lacuna na formação do discente de enfermagem nesta área do conhecimento e, por outro lado, havia um contexto oportuno para atuação interdisciplinar em conjunto com a Comissão de Controle de Infecção Hospitalar (CClH) do Hospital Universitário de Brasília (HUB).

\section{OBJ ETIVO}

Descrever a experiência vivenciada na elaboração e no desenvolvimento de um projeto de extensão de enfermagem na prevenção e controle de infecções hospitalares no Hospital Universitário de Brasília, na cidade de Brasília - DF.

\section{TRAJ ETÓRIA METODOLÓGICA}

Trata-se de um relato de experiência na área do processo de ensino-aprendizagem em enfermagem integrado à extensão universitária.

Neste trabalho são descritas as fases de elaboração e implantação do projeto de extensão de ação contínua de enfermagem, ocorrido em abril e maio de 2004 respectivamente, e sua operacionalização, de junho de 2004 a novembro de 2005.

\section{Percurso Inicial}

0 caminho inicial para construção e operacionalização do projeto de extensão de enfermagem na modalidade de ação contínua seguiu normas estabelecidas pelo DEX. Foi utilizado formulário próprio, em que constam os principais elementos de um projeto.

Posteriormente, a proposta foi submetida à análise para parecer pelas seguintes instâncias: Coordenador de Extensão da Unidade - Faculdade de Ciências da Saúde - e do Representante de Extensão do Departamento de Enfermagem. Os pareceres emitidos foram apreciados pelo Colegiado do Curso de Enfermagem para orientar a decisão dos membros da Câmara de Extensão - CEX/DEX/UnB. Além disso, recebeu visto e ciência da Direção do Hospital Universitário e do Presidente da $\mathrm{CClH}$, que vêm dando valioso apoio às intenções da proposta do projeto.

Após apreciação nas referidas instâncias, o projeto foi encaminhado à Câmara de Extensão, que emitiu parecer favorável à implantação do projeto ora apresentado e homologou a proposta. Assim, em maio de 2004, as atividades de extensão de enfermagem em conjunto com a $\mathrm{CClH} / \mathrm{HUB}$ foram iniciadas. 
Referenciais Teóricos que Subsidiaram a Elaboração do Projeto de Extensão de Enfermagem: As Práticas de Vigilância das Infecções Hospitalares no Brasil

Os avanços tecnológicos relacionados aos procedimentos invasivos, diagnósticos e terapêuticos, e o aparecimento de microorganismos multirresistentes aos antimicrobianos usados rotineiramente na prática hospitalar tornaram as infecções hospitalares (IHs) um problema de saúde pública mundial ${ }^{5}$.

No Brasil, na década de 1950 surgiram os primeiros relatos de IHs relacionados à esterilização de material hospitalar (1956) e sobre o uso indiscriminado de antibióticos (1959), que foram publicados na Revista Paulista de Hospitais 6 .

Em 1963, no Hospital Ernesto Dorneles em Porto Alegre - Rio Grande do Sul, foi criada a primeira $\mathrm{CCIH}$, representando um marco de uma iniciativa institucional na implantação de controle de infecção hospitalar $(\mathrm{ClH})^{7}$.

Mas, somente no início da década de 1970 o processo de recomendação de controle das $\mathrm{H}$ s teve seu início, com a iniciativa do Instituto Nacional de Previdência Social (INPS) aos hospitais a ele conveniados, seguido da implantação de comissões em alguns hospitais públicos de ensino ligados às universidades, como o Hospital de Clínicas da Universidade Federal de Minas Gerais (UFMG) (1978) 7,8.

Contudo, a preocupação emergente com a $\mathrm{HH}$ no país efetivamente surgiu a partir da década de 1980, com a morte do Presidente eleito Tancredo Neves, que teve sua condição clínica agravada devido à infecção. A partir desse marcante fato, incentivaramse a ações governamentais por meio de cursos, manuais e portarias.

Inicialmente, a Portaria $n^{0} 196$ do Ministério da Saúde (MS), promulgada em 24 de junho de 1983, afirmava que "todo hospital, independentemente da entidade mantenedora, porte ou especialidade, deveria constituir Comissão de Controle de Infecção Hospitalar", sendo que a implantação e a fiscalização da mesma seria de competência das Secretarias de Saúde dos Estados. E a Portaria $n^{0} 140$ do MS, publicada em 8 de abril de 1987 criava a Comissão Nacional de Controle de Infecção Hospitalar ${ }^{6,7}$.

Em 1988, por meio da Portaria $n^{0} 232$, institui-se 0 Programa Nacional de Controle de Infecção Hospitalar ligado à Secretaria Nacional de Programas Especiais do $\mathrm{MS}^{7}$. Entretanto, foi somente com a Portaria $\mathrm{n}^{0}$ 930/ 1992 do MS que se determinou a obrigatoriedade da existência, em todo hospital brasileiro, de uma Comissão de Controle de Infecção Hospitalar, segundo recomendação da Organização Mundial de Saúde (OMS).
Apesar das tentativas de implementação de controle da infecção, dados da Coordenadoria de Controle de $\mathrm{IH}$ do MS demonstraram pouca efetividade das ações, estimando-se que apenas $10 \%$ dos hospitais haviam criado comissões para esse fim $^{9}$.

Em 1998, o MS revoga a Portaria no 930/1992 e publica a Portaria $n^{0} 2.616^{10}$. Esta legislação vigente dispõe sobre as diretrizes e as normas para prevenção e controle das IHs, considerando, entre outros aspectos, as determinações da Lei $n^{0} 9.431^{11}$, de 6 de janeiro de 1997, sobre a obrigatoriedade da manutenção, pelos hospitais do país, de Programa de Controle de Infecções Hospitalares $(\mathrm{PClH})$. Para os efeitos desta lei, definiuse PCIH como "um conjunto de ações desenvolvidas deliberada e sistematicamente, com vistas à redução máxima possível da incidência e da gravidade das infecções hospitalares". 0 programa seria desenvolvido por membros executores da $\mathrm{CCIH}$, com diferente formação profissional.

Vale ressaltar que, na Portaria $n^{0} 2.616 / 98$, destaca-se que um dos membros da $\mathrm{CClH}$ deve ser, preferencialmente, um enfermeiro. Infere-se que este destaque está respaldado na expressão deste profissional como um dos agentes principais da $\mathrm{CCIH}$, que se deve à abrangência de seu conhecimento técnico-científico, atuando como elo canalizador entre os demais profissionais de saúde e, dessa forma, facilitando o desenvolvimento de ações previstas no PCIH.

Além dessas legislações, o MS publica, em 13 de outubro de 1999, a Portaria $n^{0} 1.241$, que determina que as atividades de CIH passem a ser executadas pela Gerência de Controle de Risco à Saúde da Diretoria de Serviços e Correlatos da Agência Nacional de Vigilância Sanitária (ANVISA) ${ }^{7}$.

Outra relevante iniciativa do MS foi o Estudo Brasileiro da Magnitude das Infecções Hospitalares em hospitais terciários, publicado em 1995. Foi a primeira pesquisa brasileira sobre $\mathrm{IH}$, realizada de maio a agosto de 1994 , que avaliou 8.624 pacientes com mais de 24 horas de internação. Os resultados mostraram que o tempo médio de permanência encontrado foi de 11,8 dias, mas esse número passa para 21,7 dias entre os pacientes que contraem IH. A taxa de pacientes com IH encontrada foi de $13,1 \%$, variando de $10 \%$, nos hospitais privados sem fins lucrativos, a $18,4 \%$ nos hospitais públicos. Considerou-se que essa diferença se explica pelo fato de os casos mais complexos serem encaminhados aos hospitais públicos, e que é preciso considerar que não existe uma taxa válida universalmente. Por fim, os autores reconhecem, entre outros aspectos, que cada hospital deve conhecer 0 perfil de seus pacientes, a qualidade do atendimento de sua equipe e o conjunto de microorganismos presentes que podem ser prejudiciais ${ }^{12}$. 
Já o estudo da Avaliação da Qualidade das Ações de Controle de Infecção Hospitalar em hospitais terciários, publicado em 1995, apontou que medidas de abrangência nacional deveriam ser implantadas para se corrigir o baixo desempenho das ações de $\mathrm{ClH}$ detectado nos hospitais próprios e conveniados do SUS ${ }^{13}$.

Por parte da comunidade científica, surgiu o interesse por eventos científicos voltados ao $\mathrm{ClH}$, o que fez crescer o número de estudos para aperfeiçoamento dos métodos de vigilância epidemiológica. Na segunda metade da década de 1990, houve a necessidade de se controlar a qualidade dos hospitais, que cresceram, tendo os profissionais e as instituições de saúde começado a buscar modelos operacionais ${ }^{14}$.

Em relação à produção científica nacional, desde a década de 1950, vê-se voltada predominantemente para o ambiente (procedimentos de anti-sepsia, esterilização, desinfecção, higiene, áreas de risco, fluxo de pessoal, material). Entretanto, a partir da década de 1970, destaca-se a multicausalidade, atribuindo-se as infecções não mais apenas ao ambiente, mas também à suscetibilidade individual e à introdução de novas tecnologias ${ }^{14,15}$.

Quando na década de 1980 a IH evoluiu em quantidade e gravidade, chamando a atenção dos veículos de comunicação, a produção científica manifestou-se com denúncias, evidenciando a necessidade de organização da $\mathrm{CCIH}$. Assim, iniciaramse as principais ações governamentais, validando e padronizando produtos, tais como desinfetantes, antisépticos e esterilizantes. Na década de 1990, com a implantação da $\mathrm{CCIH}$ nos hospitais, a atenção voltouse para os métodos de vigilância epidemiológica, na tentativa de prevenção e controle da $\mathbb{I}^{14,15}$.

Como produto do desenvolvimento do sistema de vigilância epidemiológica das IHs no país, foram publicadas fontes de referência orientadoras de ações de medidas de prevenção e controle de $\mathrm{IH}$, como: o Manual de Controle de Infecção em Cirurgia do Colégio Brasileiro de Cirurgiões, de autoria de Edmundo Machado Ferraz (1982); o Manual de Controle de Infecção Hospitalar pelo MS (1985); o Manual de Processamento de Artigos e Superfícies em Estabelecimentos de Saúde (MS, 1994); o Manual de Vigilância Epidemiológica por Componente - tradução do sistema National Nosocomial Infection Surveillance (NNIS) do Center for Disease Control and Prevention dos EUA (1994); e o Estudo Brasileiro da Magnitude das Infecções Hospitalares em hospitais terciários (MS, 1995). $E$, também, diversas obras literárias de eminentes estudiosos brasileiros na área foram publicadas.

A partir do início da década de 2000, a ANVISA, dentro da Gerência de Tecnologia em Serviços de Saúde
(GGTES), criou a Unidade de Controle de Infecção Hospitalar (UCISA), que, por meio da Portaria $n^{0} 385 /$ 2003, passou a ser a Gerência de Investigação e Prevenção das Infecções e dos Eventos Adversos (GIPEA). A GIPEA vem desenvolvendo diversas atividades relacionadas à pesquisa e à educação dos profissionais de saúde, referentes às ações de vigilância epidemiológica das IHs, de investigação de surtos, entre outras. A perspectiva nestes focos de trabalho é a ampliação e o fortalecimento do sistema de vigilância à saúde no país.

Considerando os relevantes fatos históricos que determinaram a implantação e os avanços das medidas de controle de $\mathrm{IH}$ no Brasil, pelas práticas de $\mathrm{ClH}$ determinadas por políticas públicas sociais na área da saúde, traçou-se a proposta do Projeto de Extensão de Enfermagem no PCIH do HUB, a seguir apresentada.

\section{Objetivos da Ação Extensionista}

Com o intuito de contribuir para a melhoria das ações previstas no $\mathrm{PClH}$ do HUB e tornar oportunas aos discentes de enfermagem vivências contextualizadas de ações de prevenção e controle de IHs, foram traçados como objetivos do projeto:

- Apoiar o Serviço de Controle de Infecção Hospitalar, especificamente com a Comissão de Controle de Infecção Hospitalar, atuando de acordo com as ações previstas no Programa de Controle de Infecção Hospitalar do Hospital Universitário de Brasília.

- Desenvolver competências técnico-científicas, atitudes e valores referentes à atuação do (a) Enfermeiro (a), permeadas por ações de prevenção e controle de infecções hospitalares e de educação em serviço.

\section{Atores e Cenário da Ação Extensionista}

0 projeto caracteriza-se pela execução interdisciplinar e interinstitucional - UnB/HUB, envolvendo os seguintes agentes: dois docentes do Curso de Enfermagem da Universidade de Brasília, coordenadores e executores do projeto; dois extensionistas voluntários em 2004 e cinco bolsistas de extensão em 2005; e os membros executores da CCIH/HUB (dois médicos, uma enfermeira, duas auxiliares de enfermagem e uma secretária).

Foram cenários de desenvolvimento das atividades os diferentes serviços da instituição hospitalar.

\section{Ações Extensionistas}

0 Quadro 1 indica as ações extensionistas a serem desenvolvidas pelos agentes executores do projeto, descritas em três etapas de operacionalização. 


\section{Quadro 1:}

Ações extensionistas de enfermagem. Brasília (DF), 2004/2005

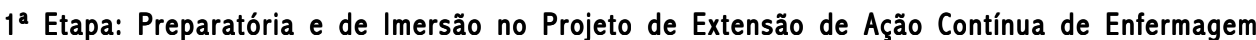

- Seleção de discentes para compor a equipe de trabalho, segundo critérios estabelecidos pela coordenação do projeto;

- Apresentação e discussão da proposta de trabalho em reunião.

2 ${ }^{\mathrm{a}}$ Etapa: Operacionalização de Enfermagem - a equipe de trabalho foi integrada à CCIH/HUB e desenvolveu as seguintes atividades: - Debate sobre o Programa de Controle de Infecção Hospitalar (PCIH) instituído no HUB;

- Participação ativa no Sistema de Vigilância Epidemiológica das Infecções Hospitalares (SVEIH), segundo método de vigilância epidemiológico adotado na instituição hospitalar;

- Realização de investigação epidemiológica de casos e surtos, sempre que indicado, e implantação de medidas imediatas de controle, segundo normas e rotinas da $\mathrm{CClH}$;

- Realização da notificação das IHs;

- Realização do monitoramento dos casos - pacientes com IH;

- Participação na elaboração do relatório mensal dos indicadores epidemiológicos interpretados e analisados;

- Participação na avaliação periódica e sistemática das informações providas do SVEIH;

- Realização de visitas técnicas nas áreas destinadas a pacientes críticos e outros setores;

- Participação na implementação do Projeto do Serviço Ambulatorial de Egressos Cirúrgicos do hospital.

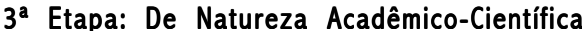

- Levantamento bibliográfico sobre a temática central infecção hospitalar;

- Elaboração de Manual de Procedimentos em Vigilância Pós-Cirúrgica;

- Elaboração de trabalho acadêmico para divulgação científica/experiência vivenciada, em periódico especializado e/ou on-line;

- Participação em reuniões científicas com a equipe de trabalho e da CCIH/HUB;

- Participação em eventos científicos.

Figura 1: Sistema e componentes do processo de avaliação do Projeto de Extensão de Ação Contínua de Enfermagem no Controle de Infecção Hospitalar do Hospital Universitário de Brasília.

\begin{tabular}{|lll}
\hline Qualitativa & CATEGORIAS DE AVALIAÇ̃̃O \\
Quartitativa
\end{tabular}

- A tividades desenvolvidas

- Integração acadêmica (ersino $\mathrm{x}$ pesquisa)

-Irtegação das áneas de combecimerto

(inter e multidisciplinariedade)

- Impacto de açã e resultados obtido

- Produtos gerados

- Avaliação feita pelos bereficiános

-Comparaz̃o das metas propos tas e os resultados akançados
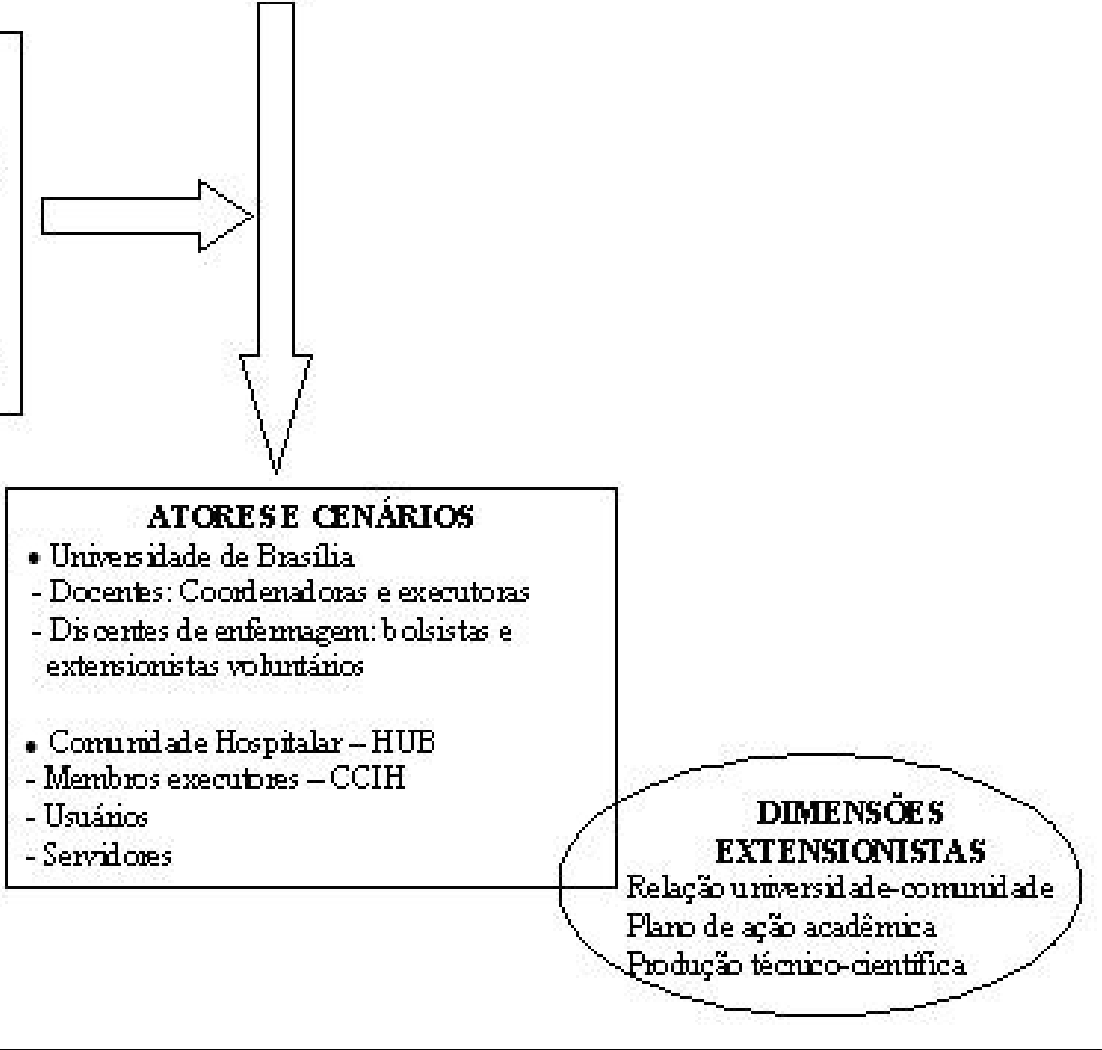


\section{Sistema e Componentes da Avaliação do Projeto}

Os componentes da avaliação do processo da ação extensionista são apresentados na Figura 1. Ressalta-se que o principal referencial da avaliação foi o comportamento da realidade como conseqüência do projeto, ou seja, de que modo foram produzidas transformações nos atores envolvidos e no contexto de desenvolvimento do projeto.

Assim, as informações providas da avaliação de cada ator envolvido, de forma individual e coletiva, foram expressas em Relatório Final elaborado anualmente, e encaminhado à Diretoria Técnica de Extensão - DTE/DEX - para fins de avaliação institucional de extensão universitária.

\section{Financiamento}

0 apoio financeiro à operacionalização das atividades de extensão foi oriundo do Programa de Apoio a Projetos de Extensão de Ação Contínua do DEX/UnB, por meio de edital de concorrência lançado anualmente.

Em 2004, o projeto foi selecionado e contemplado com recurso financeiro à obtenção de materiais de consumo e permanente, essenciais à viabilização das ações previstas. Já em 2005, recebeu apoio do Programa de Bolsas de Extensão e de Estudantes Extensionistas Voluntários, a fim de viabilizar a participação do estudante de enfermagem no projeto de forma remunerada.

\section{RESULTADOS}

As experiências vivenciadas, percebidas e sentidas no cotidiano do desenvolvimento das ações extensionistas de enfermagem, pelas docentes cooordenadoras-executoras, dos bolsistas de extensão e dos extensionistas voluntários, juntamente com os componentes da $\mathrm{CClH} / \mathrm{HUB}$, ocorreram permeadas pelo saber-fazer e saber-ser construtivista e colaborativo.

Desse modo, as seguintes ações de enfermagem foram realizadas: reconhecimento do funcionamento e rotina do Serviço de Controle de Infecção Hospitalar da instituição $(\mathrm{SClH})$; conhecimento do $\mathrm{PCIH}$ do HUB, debatendo especificamente sobre a atuação do(a) enfermeiro(a) na vigilância epidemiológica das IHs; leitura de textos acerca da legislação brasileira em controle de $\mathrm{IH}$ e de outros referenciais teóricos, com momentos de discussão para ensino e aprendizagem; participação no "Curso de Controle de Infecção Hospitalar para Residentes" oferecido pela $\mathrm{CClH} / \mathrm{HUB}$; imersão na prática, realizando a busca ativa de casos de IH na Unidade de Clínica Médica, Clínica Cirúrgica, Pronto Socorro, Unidade de Tratamento Intensivo, Maternidade e Berçário; notificação dos casos diagnosticados como infecção hospitalar ou infeç̧ão comunitária - total de 67 notificações; realização de monitoramento de casos diagnosticados, implantando medidas de controle segundo normas e rotinas estabelecidas pela $\mathrm{CCIH}$; auxílio ao $\mathrm{SClH}$ no cálculo das taxas de $\mathrm{IH}$ do hospital, utilizando o Programa Excel ${ }^{\circledR}$; colaboração na interpretação e na análise dos dados providos pelo Sistema de Vigilância de Infecção Hospitalar (SVIH), auxiliando na elaboração de relatório mensal dos indicadores epidemiológicos; realização de visitas técnicas no Centro de Material e Esterilização, Centro Cirúrgico, Centro Obstétrico, Lavanderia e Laboratório do hospital; implantação, em março de 2005, do Programa de Vigilância Cirúrgica Pós-alta Hospitalar - atividade de acompanhamento de pacientes da cirurgia geral até $030^{\circ}$ dia pós-operatório, na sala de egressos, com vistas ao seguimento da vigilância de ocorrência de infecção de sítio cirúrgico (ISC); elaboração do Manual do Serviço de Acompanhamento Ambulatorial de Egressos Cirúrgicos (SAAEC), estabelecendo normas, rotinas e procedimentos a serem realizados pelo serviço; seguimento, de março a dezembro de 2005, de 685 pacientes na sala de egressos cirúrgicos, sendo notificados 76 casos de ISC; participação, em outubro de 2005, da V Semana de Extensão da UnB, com apresentação do projeto à comunidade participante do evento.

$\mathrm{Na}$ avaliação feita conjuntamente, considerando-se as intenções do projeto e o que de concreto se realizou, os seguintes aspectos foram ressaltados como positivos: inserção do discente de enfermagem na $\mathrm{CClH}$; acolhimento pelos membros do Serviço de Controle de Infecção Hospitalar (SCIH); co-participação dos funcionários da $\mathrm{CClH}$ no projeto; desenvolvimento de atividades específicas de atuação do(a) enfermeiro(a) na prevenção e controle de $\mathrm{IH}$; fortalecimento da integração entre a Universidade e a comunidade hospitalar; aproximação do discente de enfermagem com a produção técnico-científica, por meio de vivências práticas.

Os aspectos julgados como necessários a serem fortalecidos foram apontados e discutidos, firmandose a relevância de se dar continuidade ao projeto com algumas inovações. Assim, a proposta do projeto foi renovada para o ano de 2006 com a concessão de bolsas de extensão, fortalecendo as ações já previstas na proposta inicial e ampliando para outras ações tais quais: implantação de sistema de vigilância pós-alta a pacientes submetidas a cesarianas no hospital; atuação contínua no ambulatório de egressos cirúrgicos; revisão do manual do SAAEC; elaboração e desenvolvimento de projeto de pesquisa sobre incidência de infecção de sítio cirúrgico (ISC) em pacientes submetidos a herniorrafia; elaboração de proposta de atividade de educação em serviço sobre medidas de prevenção e controle de $\mathrm{HH}$. 


\section{CONCLUSÃO}

Considerando a magnitude e complexidade que envolve o processo de trabalho para prevenção e controle de infecções hospitalares na assistência à saúde nos diversos microcontextos de uma instituição hospitalar, assim como a relevância acerca do preparo inicial de discentes neste campo do saber-fazer e saber-ser, nos é permitido afirmar que a implantação e execução do Projeto de Extensão 'Atuação de Enfermagem no Programa de Controle de Infecção Hospitalar do Hospital Universitário de Brasilia' trouxe benefícios para alunos, professoras e membros executores da $\mathrm{CClH} / \mathrm{HUB}$, assim como para a comunidade do hospital-escola.

Em relação às vivências de ensino-aprendizagem, as atividades de extensão desenvolvidas ocorreram como estratégia de ensino-aplicação, como Loyola e Oliveira ${ }^{16: 432}$ afirmam:

É na extensão que as enfermeiras vão entender $e$ fundamentar os conceitos e teorias aprendidos nas atividades de ensino, consolidando $e$ complementando o aprendizado com aplicação. Este é um dos grandes méritos da extensão, o de permitir a efetivação do aprendizado com aplicação.

Focalizando uma análise na perspectiva da comunidade hospitalar envolvida, pode-se inferir que, neste cenário, houve alguns avanços, na medida em que se fortaleceu a prática assistencial, empenhada em oferecer cuidados aos usuários do Sistema Único de Saúde do Hospital Universitário de Brasília, pautados no aprimoramento técnico-científico, especificamente no controle de infecções hospitalares.

\section{Referências}

1. Faria DS. Extensão universitária: do assistencialismo à autonomia, do mercado ao social. Rev Dec Ext UnB 1999; 3(4): 14-6.

2. Ministério da Educação (BR). Fórum de Pró-Reitores de Extensão das Universidades Públicas Brasileiras. Avaliação nacional da extensão universitária. Brasília (DF): MEC/SESu; Paraná (PR): UFPR; llhéus (BA): UESC; 2001.

3. Ministério da Educação (BR). Fórum de Pró-Reitores de Extensão das Universidades Públicas Brasileiras e SESu/MEC. Plano Nacional de Extensão Universitária. [online] 2000/2001; [citado 3 out 2005]; [aprox 16 telas]. Disponível em: http://www.renex.org.br/ arquivos/pne/mapa.htm.

4. Universidade de Brasília-UNB. A extensão na Universidade de Brasília: o que é e como participar. Brasília (DF); 2004.
Assim, considerando o contexto interdisciplinar e interinstitucional em que a proposta foi idealizada e concretizada, pode-se considerar que o projeto de ação contínua de enfermagem no controle de $\mathrm{H}$ :

- se desenvolveu como trabalho processual, onde houve interação universidade-professorasalunos-comunidade hospitalar, ocorrendo mútua modificação e complementaridade nos sujeitos-ação, na perspectiva de oferecer uma assistência à saúde voltada à realidade e necessidades dos sujeitos-alvo;

- se constituiu de estratégia efetiva de aprendizado mútuo e de realização de ações simultaneamente transformadoras entre Universidade e comunidade hospitalar;

- propiciou a formação de profissionais-cidadãos capazes de discutir e apresentar propostas às questões relacionadas ao controle de infecções hospitalares;

- serviu como veículo de comunicação permanente, aprimorando a interlocução entre os setores Universidade/comunidade hospitalar;

- oportunizou a vivência social, política e profissional entre professoras, alunos, profissionais da área da saúde, técnico-administrativos e, principalmente, dos usuários do Sistema de Saúde do Hospital Universitário de Brasilia.

Concluindo, essa experiência de extensão universitária oportunizou a troca mútua de saberes, uma vez que o planejamento e a execução da proposta do projeto possibilitaram e promoveram a aproximação da Universidade de Brasília - Curso de Enfermagem - com a comunidade do Hospital Universitário de Brasília, e, dessa forma, alguns muros (in) visíveis foram ultrapassados.

5. Turrini RNT, Santo AH. Infecção hospitalar e causas múltiplas de morte. J Ped [online] 2002; [citado $20 \mathrm{dez} 2004$ ] 78(6): [aprox. 9 telas]. Disponível em: http://www.scielo.br

6. Rodrigues EAC. Histórico das infecções hospitalares. In: Rodrigues EAC, Mendonça JS, Amarante JMB. Infecções hospitalares: prevenção e controle. São Paulo (SP): Savier ; 1997.

7. Martins MA. Aspectos históricos da infecção hospitalar. In: Oliveira AC, Armond GA, Clemente WT, editores. Infecções hospitalares: epidemiologia, prevenção e controle. Rio de Janeiro (RJ): Guanabara Koogan; 2005.

8. Oliveira AC, Rocha LC. Prevenção das infecções hospitalares cirúrgicas. In: Oliveira AC, Albuquerque CP, Rocha LC. Abordagem das infecções hospitalares: prevenção e controle. Rio de Janeiro (RJ): MEDSI; 1997. 
14. Poveda VB. Análise dos fatores predisponentes à infecção do sítio cirúrgico em gastrectomia [dissertação de mestrado] Ribeirão Preto (SP): Escola de Enfermagem de Ribeirão Preto/ USP; 2004.

15. Lacerda RA. Produção científica nacional sobre infecção hospitalar e a contribuição da enfermagem: ontem, hoje e perspectivas. Rev Latino-Am Enfermagem 2002; 10(1): 55-63.

16. Loyola CMD, Oliveira RMP. A universidade "extendida": estratégias de ensino e aprendizagem em enfermagem. Esc Anna Nery Rev Enferm 2005 dez; 9(3): 429-33.

\section{Sobre a Autora}

\section{Maria Cristina Soares Rodrigues}

Enfermeira e Farmacêutica-Bioquímica. Doutora em Ciências da Saúde. Professora Adjunto do Departamento de Enfermagem da Faculdade de Ciências da Saúde da UnB. Pesquisadora do Núcleo de Estudos em Enfermagem, Educação e Processo de Trabalho em Saúde, Ambiente e Vigilância - NEEPTS. Coordenadora do Projeto de Extensão de Ação Contínua "Atuação de Enfermagem no Programa de Controle de Infecção Hospitalar do Hospital Universitário de Brasília". Universidade de Brasília (UnB). Brasília-DF. 